\title{
Strategies for Combating Systematics at LHCb
}

\author{
Guy Wilkinson \\ University of Oxford, Denys Wilkinson Building, Keble Road, Oxford, OX1 3RH, UK \\ for the $\mathrm{LHCb}$ collaboration
}

Contribution to 'Beauty 2005', June 2005, Assisi, Italy

\begin{abstract}
It is expected that $\mathrm{LHCb}$ will make many measurements of $\mathrm{CP}$ violating observables with excellent statistical precision. Good control of systematic effects is therefore essential. It is discussed how this challenge will be met. Emphasis is given to the problem of measuring the flavour tagging performance. A summary is also presented of the output streams which will be used to calibrate the proper time resolution and particle identification.
\end{abstract}




\section{Introduction}

LHCb will accumulate very high statistics in a wide range of channels sensitive to CP violation. For example, over 200k triggered and reconstructed events will be accumulated in one nominal year of operation in the channel $B^{0} \rightarrow J / \psi(\mu \mu) K_{S}^{0}$ [1]. In order not to compromise this statistical precision, excellent systematic control is required.

Systematic challenges must be overcome in many areas. Here preliminary studies are presented which address the problem of measuring the flavour tagging performance, an essential ingredient in time-dependent $\mathrm{CP}$ asymmetry measurements. A brief summary is also given of the high statistics control channel streams which will be output by the high level trigger, in order to help with other calibration tasks.

\section{Calibration of the Flavour Tagging}

\subsection{Control Channels}

In measuring a time-dependent $\mathrm{CP}$ asymmetry it is necessary to know whether the initial state of the meson of interest contained a $b$ or a $\bar{b}$ quark. The procedure by which this is done is known as flavour tagging. In LHCb flavour tagging is performed through a combination of several methods. These include leptons and kaons from the 'other' B in the event, 'same side' pions (kaons) associated with the fragmentation process which produced the $B^{0}\left(B_{s}^{0}\right)$ of interest, and vertex and jet charge tags.

In practice, flavour tagging is not perfect, and mistags will occur with some probability, usually defined as $\omega$. The consequence of mistags is that the observed asymmetry is diluted by a factor $(1-2 \omega)$ with respect to the true asymmetry. In order to extract the underlying physics it is thus necessary to know the value of $\omega$. Given the expected statistical precision on the $\mathrm{CP}$ asymmetries for the most important channels, it can be estimated that $\omega$ must be known with a relative precision of better than $1 \%$ in order not to contribute a dominant error.

The mistag rate can be measured directly from control channels with a flavour specific final state. These include $B^{+} \rightarrow J / \psi K^{+}, B^{0} \rightarrow J / \psi K^{* 0}$ and $B_{s}^{0} \rightarrow D_{s}^{+} \pi^{-}$. However, in simulation studies the values of the mistags measured on these control channels are found to differ from each other, and from those values measured on signal channels using truth information. A selection of these mistag rates is listed in Table 1, with statistical errors. There is a significant variation in performance which is large compared to the desired precision. This variation is however expected, and arises because of correlations between the trigger and tagging.

\begin{tabular}{ll}
\hline Channel & Mistag rate \\
\hline$B^{0} \rightarrow \pi \pi$ & $0.358 \pm 0.002$ \\
$B^{+} \rightarrow J / \psi K^{+}$ & $0.377 \pm 0.002$ \\
$B_{s} \rightarrow J / \psi \phi$ & $0.346 \pm 0.002$ \\
$B_{s} \rightarrow D_{s} \pi$ & $0.323 \pm 0.005$ \\
\hline
\end{tabular}

Table 1: Mistag rates in selected channels. 


\subsection{Correlations with the LHCb Trigger}

The LHCb trigger system is described in detail elsewhere [2]. Here the most essential components are summarised. At the earliest level of triggering, L0, the event is accepted if a single high transverse momentum $\left(p_{t}\right)$ muon, electron or hadron is detected. The next stage, L1, relies primarily on lifetime information: a positive decision is given if two or more tracks are found with a significant impact parameter, and some non-negligible transverse momentum. High mass di-muons also fire L1.

In a given event of interest the trigger can fire on the signal decay itself, or the other decaying $\mathrm{B}$ hadron. These different possibilities have consequences for the tagging. Compare, for example, $B_{s}^{0} \rightarrow J / \psi \phi$ and a $B_{s}^{0} \rightarrow D_{s} \pi$. The sample of L0 accepted events for the former mode will be dominated by decays in which the trigger fired on a lepton from the $J / \psi$. In the hadronic mode, the overall efficiency will be lower, with the high $p_{t}$ hadron trigger most likely to select the event. However, there will also be events triggered by a lepton from the other $\mathrm{B}$ hadron in the event, and this fraction of triggers will be significantly greater than in the $B_{s}^{0} \rightarrow J / \psi \phi$ case. This category of triggers provides a high quality flavour tag, and hence a lower mistag rate is expected for selected $B_{s}^{0} \rightarrow D_{s} \pi$ events, consistent with what is seen in Table 1. A variation in tagging performance is also expected depending on how the event is triggered at L1. For events where a non-signal $B^{0}$ fires the L1 lifetime trigger, the bias to longer lifetimes will lead to a greater probability of this B having oscillated. The tagging performance in this sample will be degraded.

In order to apply the measurement from a control channel to a $\mathrm{CP}$ mode it is therefore necessary to ensure that the events have been triggered in an identical manner. This can be done by evaluating the performance in two categories ${ }^{1}$ :

- Trigger on Signal (TOS): events where the trigger fires on particles from the signal decay alone;

- Trigger Independent of Signal (TIS): events where the trigger fires on particles associated with the other B decay, or indeed from the underlying event.

As it is necessary to distinguish between the two trigger levels, four classes must be considered: 'LO TIS, L1 TIS', 'LO TIS, L1 TOS', 'LO TOS, L1 TIS' and 'LO TIS, L1 TOS'. It may be hypothesised that the tagging performance is independent of the signal decay mode in each of these four classes.

In order to test this hypothesis, a high statistics generator study has been performed in which the essential aspects of the trigger system and tagging algorithm have been simulated. The analysis has been performed for $B_{s}^{0} \rightarrow D_{s} \pi$ as a control channel and $B_{s}^{0} \rightarrow J / \psi \phi$ as the CP channel. The mistag rate of all triggered events was measured to be $0.2463 \pm 0.0007$ and $0.2705 \pm 0.0006$ for the control and CP channels respectively, showing a similar difference to that observed in Table 1. (The absolute values differ because of the simpler treatment implemented in the generator study.) Figure 1 shows the mistag rate subdivided into the four TIS/TOS classes. As expected there is a significant difference in performance between each category, and for the two 'LO TIS' classes there is excellent agreement between the two channels, supporting the hypothesis. In the other two classes, however, there is no agreement.

\footnotetext{
${ }^{1}$ Note that the L1 trigger involves two tracks, and has the possibility to fire on a mixture of information from the signal decay and elsewhere. This is a third category which is omitted in the present discussion.
} 


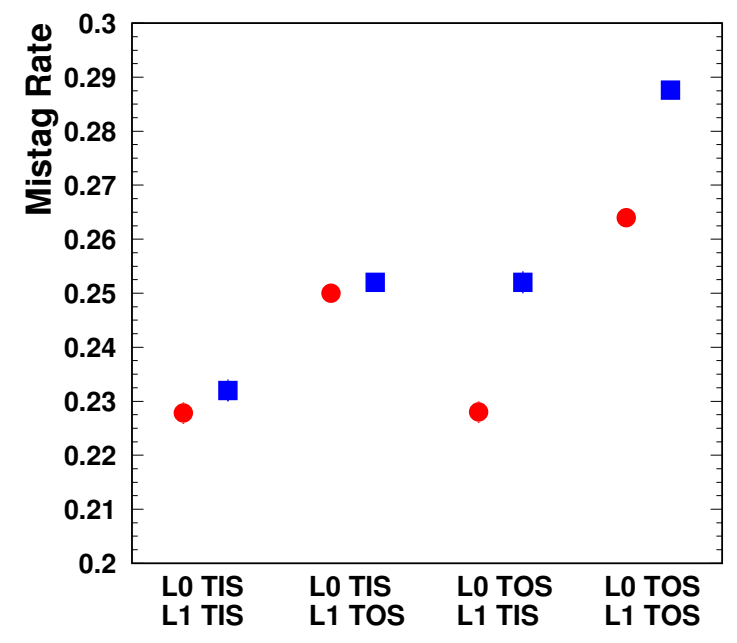

Figure 1: Mistag rate for $B_{s}^{0} \rightarrow D_{s} \pi$ (circles) and $B_{s}^{0} \rightarrow J / \psi \phi$ (squares), subdivided into TIS/TOS categories. (Results from generator studies.)

\subsection{Kinematical Correlations}

The disagreement in mistag between the two channels in the categories involving 'LO TOS' can be understood as arising from the correlation in kinematics between the two b hadrons. The L0 trigger biases the kinematics of the signal meson it selects, through the $p_{t}$ requirement, which is different for the hadron and muon triggers. For this reason the kinematics of the signal mesons in the 'LO TOS' classes are different for the two decays. The kinematics of the two hadrons in the event are correlated, and hence the $p_{t}$ of the tagging hadron in the two modes differs, even though the trigger fired on the signal B. As the quality of a tag varies with the $p_{t}$ of the tagging particle, and therefore with the $p_{t}$ of the decaying hadron, a difference in mistag rates arises. (A similar bias can occur from the offline selection cuts.)

This explanation may be tested in each of the two 'LO TOS' categories by measuring the mistag rate of the control channel in bins of the signal hadron $p_{t}$. The normalised signal $p_{t}$ spectrum of the $\mathrm{CP}$ channel is measured from the data, and the mistag rate from the control channel is applied bin-by-bin to this spectrum. In this manner the control channel tagging performance is re-weighted to reproduce the kinematics of the signal hadron in the CP channel. As the kinematics of the signal and tagging hadron are correlated, this procedure is expected to result in an identical tagging performance. After this reweighting agreement is also expected for the tagging hadron kinematics. Figure 2 shows the tagging performance in the four trigger classes, after reweighting. Satisfactory agreement is now seen in all categories, with a maximum discrepancy of two sigma present in the 'LO TOS, L1 TIS' category.

\subsection{Application to the Data}

The studies performed at generator level indicate that it is possible to evaluate a tagging performance from control channels in a manner which can be applied to the CP channels. This procedure, however, relies on the assumption that it is possible to distinguish between the 'TOS' 


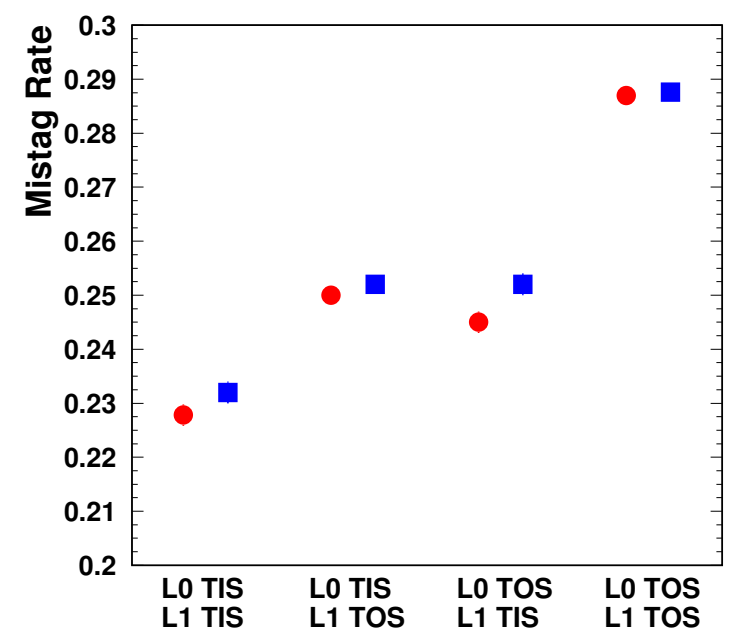

Figure 2: Mistag rate for $B_{s}^{0} \rightarrow D_{s} \pi$ (circles) and $B_{s}^{0} \rightarrow J / \psi \phi$ (squares) after $p_{t}$ reweighting. (Results from generator studies.)

and 'TIS' categories and understand what particles fired the trigger. In the generator study this category separation was performed by accessing 'truth' information.

A method known as 'buffer tampering' has been developed which allows this assignment of the trigger category to be made without inspecting the truth information. The association between the hits in the raw data buffer and a given offline track are known. These hits can be masked and the trigger algorithm re-run on the event in the offline analysis, as if that particle did not exist. By removing suitable combinations of tracks in turn it is possible to decide which of the four trigger categories the event falls into.

Figure 3 presents the results of a preliminary study performed using full simulation and the buffer tampering tool. Mistag rates are shown in the four trigger categories, after $p_{t}$ reweighting has been applied. As was the case in the generator study, there is consistency between the control and $\mathrm{CP}$ channels, although higher statistics are required to draw firm conclusions.

\section{Lighthouse HLT Streams}

The flavour tagging is only one aspect of the LHCb performance which will need to be properly calibrated in order to ensure a successful analysis. The finite proper time resolution dilutes the observed CP asymmetry in the $B_{s}^{0}$ channels in a similar manner to the flavour tagging, and hence must be well understood. The particle identification (PID) provided by the RICH system is vital in suppressing backgrounds from specific decay topologies which peak close to the signal. The PID must be well calibrated in order to be able to estimate the residual contamination. High rate 'lighthouse' streams in the high level trigger (HLT) will be used to meet these and related challenges. These streams are as follows:

- Lifetime unbiased di-muons will be written out at a rate of $600 \mathrm{~Hz}$ based on a selection involving invariant mass alone. The prompt $J / \psi$ component of this stream will be 


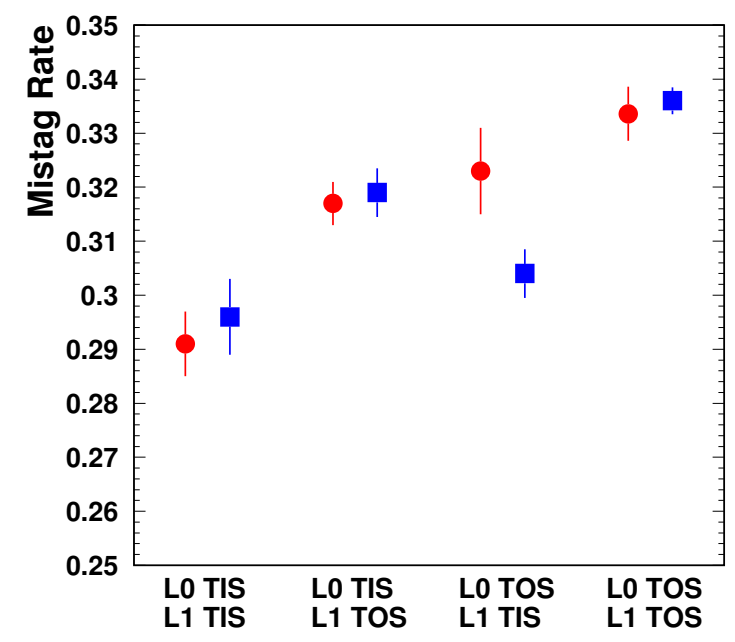

Figure 3: Mistag rate for $B_{s}^{0} \rightarrow D_{s} \pi$ (circles) and $B_{s}^{0} \rightarrow J / \psi \phi$ (squares), after $p_{t}$ reweighting. (Results from full simulation.)

invaluable in calibrating the vertex and proper time resolution.

- Kinematically selected $D^{*}$ events will be allocated an output rate of $300 \mathrm{~Hz}$. These decays will provide a high purity sample of kaons and pions for use in the particle identification calibration.

- Single muons with significant impact parameter will be selected at a rate of $900 \mathrm{HZ}$. These will provide a large sample of B hadrons which can be used to understand biases introduced by other components of the trigger system.

These triggers will operate in parallel with the $200 \mathrm{~Hz}$ selection which is dedicated to the exclusive B decay channels of the core physics programme.

\section{Conclusions}

Significant attention is now being paid to the challenge of systematic control in LHCb analyses. A key problem is the calibration of the flavour tagging performance, where correlations between the trigger and tagging lead to systematic differences in the mistag rates between the $\mathrm{CP}$ channel values and the control channel measurements. A procedure has been established which allows these biases to be eliminated. Other challenges will be tackled through the use of very high statistics 'lighthouse channel' streams included in the output of the high level trigger.

\section{References}

[1] LHCb Collaboration, Reoptimzed detector design and performance, LHCC-2003-030.

[2] Frederic Teubert, The LHCb Trigger System, these proceedings. 